\title{
Heavy Quark Mass Effects in PQCD and Heavy Flavor Parton Distributions
}

\author{
Wu-Ki Tung ${ }^{1,2}$, H.L. Lai ${ }^{1,2,3}$, J. Pumplin ${ }^{1}$, P. Nadolsky ${ }^{4}$, and C.-P. Yuan ${ }^{1}$. \\ ${ }^{1}$ Michigan State University, East Lansing, MI - USA \\ ${ }^{2}$ University of Washington, Seattle, Washington - USA \\ 3 Taipei Municipal University of Education, Taipei, Taiwan \\ ${ }^{4}$ Argonne National Laboratory, Argonne, IL, USA
}

\begin{abstract}
The systematic treatment of heavy quark mass effects in DIS in current CTEQ global analysis is summarized. Applications of this treatment to the comparison between theory and experimental data on DIS charm production are described. The possibility of intrinsic charm in the nucleon is studied. The issue of determining the charm mass in global analysis is discussed.
\end{abstract}

\section{Introduction}

Contemporary global QCD analyses of high precision Deep Inelastic Scattering (DIS) data, along with other hard processes, require a consistent treatment of heavy quark mass effects in the perturbative QCD (PQCD) framework. This review [1] summarizes key features of the formalism implemented in the current CTEQ global analysis project [3] and results on its application to heavy flavor physics in global analysis [2]. Sec. 3 presents the results of the new global fits compared to heavy flavor production data in DIS [3]. Sec. 4 addresses issues related to possible intrinsic charm in the nucleon [6]. Sec. 4 discusses the topical question: can the charm mass be reliably determined in global QCD analysis?

Due to space limitation, it is impossible to include in this short written report the figures that illustrate the results discussed in the corresponding talk, as summarized above. However, since the slides for the talk have been made available at the official conference URL [1], we shall make use of these, and refer the reader to the actual figures by the slide numbers where they appear in the posted talk [1]. The same space limitation restricts citations to only the papers and talks on which this report is directly based.

\section{General PQCD framework including heavy quark masses}

The key features of the general-mass PQCD framework of [3] is illustrated in slide 3 of [1]. Factorization Formula and (scheme-dependent) summation over parton flavors: Collins has established that the PQCD factorization theorem for the structure functions takes the general form $F_{\lambda}\left(x, Q^{2}\right)=\sum_{a} f^{a} \otimes \widehat{\omega}_{a}^{\lambda}$ even when the heavy quark mass effects are kept. Here, the summation is over the active parton flavor label $a, f^{a}(x, \mu)$ are the parton distributions at the factorization scale $\mu$, and $\widehat{\omega}_{a}^{\lambda}\left(x, Q / \mu, M_{i} / \mu\right)$ are the infrared safe Wilson coefficients (or hard-scattering amplitudes) that can be calculated order-by-order in perturbation theory. The summation over "parton flavor" label $a$ in the factorization formula is determined by the factorization scheme chosen to define the parton distributions $f^{a}(x, \mu)$. In general, we use the variable flavor number scheme.

The summation over (physical) final-state flavors: For total inclusive structure functions, the factorization formula contains an implicit summation over all possible quark flavors 
in the final state: $\hat{\omega}_{a}=\sum_{b} \hat{\omega}_{a}^{b}$, where " $b$ " denotes final state flavors, and $\hat{\omega}_{a}^{b}$ is the perturbatively calculable hard cross section for an incoming parton " $a$ " to produce a final state containing flavor " $b$ ". It is important to emphasize that " $b$ " labels quark flavors that can be produced physically in the final state; it is not a parton label in the sense of initialstate parton flavors described in the previous subsection. In a proper implementation of the general-mass (GM) formalism, the distinction between the initial-state and final-state summations must be unambiguously and correctly observed.

Kinematic constraints and rescaling: Kinematic constraints from the phase space treatment have a significant impact on the numerical results of the calculation. In DIS, with heavy flavor produced in the final state, the most natural way to ensure the correct kinematics for both NC and CC processes is to use the rescaling variable $\chi=x\left(1+\left(\Sigma_{f} M_{f} / Q\right)^{2}\right)$ in place of the usual Bjorken $x$ in the convolution integral of the factorization formula. Here $\Sigma_{f} M_{f}$ is the sum of all heavy flavor masses in the final state. This is the ACOT $\chi$ prescription used in most recent literature.

Hard Scattering Amplitudes and the SACOT Scheme: The hard scattering amplitude $\widehat{\omega}_{a}^{\lambda}\left(x, Q / \mu, M_{i} / \mu\right)$ is by definition infrared safe, meaning it is free from logarithmic "mass-singularities" in the limit $M_{i} / Q \rightarrow 0$. Within the PQCD formalism, there is some freedom to choose how the finite mass effects are treated. The choice that makes the calculation simplest while retaining full accuracy (the SACOT scheme) can be stated as: keep the heavy quark mass dependence in the Wilson coefficients for partonic subprocesses with only light initial state partons $(g, u, d, s)$; but use the zero-mass Wilson coefficients for subprocesses that have an initial state heavy quark $(c, b)$. For the 4-flavor scheme to order $\alpha_{s}$ (NLO), we do the following: (a) keep the full $M_{c}$ dependence of the gluon fusion subprocess; (b) for NC scattering $(\gamma / Z$ exchanges), set all quark masses to zero in the quark-initiated subprocesses; and (c) for CC scattering ( $W^{ \pm}$exchange), set the initial-state quark masses to zero, but keep the final-state quark masses on shell.

Choice of Factorization Scale: The total inclusive structure function $F_{i}^{\text {tot }}$ is infrared safe. Consider the simple case of just one effective heavy flavor charm, $F_{i}^{\text {tot }}=F_{i}^{\text {light }}+F_{i}^{c}$ for any given flavor-number scheme. Since the right-hand side of this equation is dominated by the light-flavor term $F_{i}^{\text {light }}$, and the natural choice of scale for this term is $\mu=Q$, it is reasonable to use this choice for both terms to ensure infrared safety. On the other hand, in the case of experimentally measured semi-inclusive DIS structure functions for producing a charm particle in the final state, $F_{i}^{c}$ is theoretically infrared unsafe beyond NLO. One may nonetheless perform comparison of NLO theory with experiment with the understanding that the results are intrinsically less reliable, and they can be sensitive to the choice of parameters. The most natural choice of factorization scale in this case is $\mu=\sqrt{Q^{2}+M_{c}^{2}}$.

\section{Results and Comparison with heavy flavor production data}

Slides 4 and 5 of [1] show the size of heavy quark mass effects on the calculation of $F_{2}(x, Q)$ and $F_{L}(x, Q)$. The color coded areas (with complementary contours) indicate the fractional differences between GM and zero-mass (ZM) calculations. Understandably, the largest differences occur at low $Q$ and low $x$; and the significance is much more for $F_{L}(x, Q)$ than for $F_{2}(x, Q)$, since the former vanishes at LO for the ZM case. As indicated in slide 6, the GM calculation is stable and robust. It has been used as the basis for a new round of global analysis of PDFs, using the full set of HERA Run I neutral current (NC) and charged current (CC) total cross section and heavy flavor production data, along with the usual DY 
and jet data cf. [2]. Here we shall only present the comparison of the new fits to the heavy flavor production data measured at HERA.

Slide 10 shows the comparison of the ZEUS 1996-97 and 1998-2000 charm production data to the theory values obtained with the new PDF sets CTEQ6.5M (same shape for strange and non-strange seas, [3]), CTEQ6.5S0 (independent shapes for strange and nonstrange seas, [5]) as well as for the older CTEQ6HQ. Plotted are ratios of $F_{2}^{c}(x, Q)$ to that of a best fit to the respective data set. The fits to data are all reasonable. The new PDFs give slightly better fits than the previous one. Slide 11 shows the comparison of the H1 charm and bottom production data to the theory values from the same PDF sets. The $F_{2}^{c}(x, Q)$ data points have more scatter around the (smooth) theory values. The overall $\chi^{2}$ of these fits is however acceptable.

It is worth noting that correlated systematical errors are always taken into account in our global analysis. The data points shown on these plots have been shifted by the fitted systematic errors; hence the differences between the data points and the theory values as they appear on these plots give a faithful indication of the quality of the fits.

\section{Is there intrinsic charm in the nucleon?}

Many nonperturbative models of nucleon structure suggest the existence of intrinsic charm (IC) - a non-vanishing component of nucleon parton structure at the scale of $M_{c}$. On the other hand, practically all global analysis of the parton structure of the nucleon so far ignore this possibility and make the simplifying assumption that all heavy quark partons are radiatively generated: they only arise from perturbative QCD evolution, starting from zero at $\mu \sim M_{c}$. Where does the truth lie? The resolution of this dichotomy is of inherent physics interest because it concerns the fundamental structure of matter, as well as of practical interest because the cross sections for many beyond-the-standard-model (BSM) processes at hadron colliders depend on the charm parton content of the nucleon. We have addressed this problem phenomenologically by a careful global analysis based on the GM PQCD formalism that, for the first time, allows for an independent charm sector [6].

As indicated in slide 14 of [1], the following specific scenarios for the charm sea, $c(x, \mu=$ $M_{c}$ ), are explored within our GM global analysis framework: (i) the conventional radiatively generated charm; (ii) non-vanishing IC $c\left(x, M_{c}\right)$ that is sea-like (i.e. shaped as the light sea quarks); and (iii) IC of the kind suggested by light-cone wave function models of the nucleon (peaked at moderately large $x$ ). Within scenario (iii), we further distinguish two models: the one studied by Brodsky et al. (the BHPS model), and a meson cloud model.

Slide 15 summarizes the main results. The figure shows the goodness-of-fit for the global analysis, $\chi_{\text {global }}^{2}$, as a function of the magnitude of the IC component, measured by the momentum fraction carried $\langle x\rangle_{c+\bar{c}}$, under the various scenarios. In the range $0<\langle x\rangle_{c+\bar{c}}<$ 0.01 (outlined by the horizontal oval), $\chi_{\text {global }}^{2}$ is largely insensitive to $\langle x\rangle_{c+\bar{c}}$, indicating that there is no strong evidence for or against IC of a magnitude in this range. However, outside this range, for $\langle x\rangle_{c+\bar{c}}>0.01$ (outlined by the vertical oval), we see a precipitous rise of $\chi_{\text {global }}^{2}$ as $\langle x\rangle_{c+\bar{c}}$ increases. Thus our global analysis sets a useful upper bound on the amount of intrinsic charm that is consistent with existing data. Using a 90\% confidence level (C.L.) criterion, this bound is $\langle x\rangle_{c+\bar{c}}<0.02$.

Although models of IC generally do not predict $\langle x\rangle_{c+\bar{c}}$, typical guesstimates place it around 0.01 . This is consistent with the bound we determined from the above global analysis. The presence of IC of such a magnitude can have an impact on certain BSM processes, such 
as charged Higgs production in hadron collider phenomenology. Cf. slide 7 of [2], and [4, 5]. Slide 16 shows the charm distribution $c(x, \mu)$ at three energy scales $\mu=1.3,3.16,85 \mathrm{GeV}$ in the BHPS scenario, for various magnitudes of the initial distribution. We see the radiatively generated component (peaked at small $x$ ) catching up with the IC component (peaked at moderate $x$ ) as $\mu$ increases. However, the latter clearly still dominates in the $x$ region $\geqq 0.1$ even at the $\mathrm{W} / \mathrm{Z}$ mass scale.

\section{Can the charm mass be determined in global analysis?}

In principle, heavy quark masses $M_{i}(\mu)$ at some renormalization scale $\mu$ are basic parameters of QCD, similar to the coupling $\alpha_{s}(\mu)$. Thus, just like for $\alpha_{s}$, there has been recent interest in determining $M_{i}$, e.g. the charm mass $M_{c}$, from global QCD analysis. In particular, is it possible to perform a conventional global QCD analysis using $M_{c}$ as one of the fitting parameters, and thereby determine the charm mass to be the one that gives the best fit? If so, one may further ask, is this mass the $\overline{M S}$-mass or the pole-mass?

Slides 18-20 show results of a study, following the above procedure literally: one finds that the global analysis favors a relatively small values of $M_{c} \sim 1.3 \mathrm{GeV}$, and the goodnessof-fit $\chi_{\text {global }}^{2}$ increases with $M_{c}$. But, a closer examination of the problem immediately raises the question: what is the physical meaning of this favored value of $M_{c}$ ? The problem is, a chosen value of $M_{c}$ affects the global QCD analysis in two distinct ways: (i) through the mass-dependent Wilson coefficients in the theoretical calculation (the pole-mass); and (ii) through the initial condition $c\left(x, \mu=M_{c}\right)=0$ - the implicit assumption of radiatively generated charm that is used in all existing global analyses. It turns out, the global fit is influenced much more by the latter than by the former. Since radiatively generated charm is only an assumption, not an integral part of the QCD theory, the value of $M_{c}$ favored by global analysis is not directly related to the basic QCD charm mass parameter - it is neither the $\overline{M S}$-mass nor the pole-mass! In order to answer the original question "can the charm mass be determined in global analysis?", one needs to clearly differentiate between the two sources of dependence on $M_{c}$ mentioned above. This is currently under study.

\section{References}

[1] This review is based on the talk of Wu-Ki Tung in the Heavy Flavor workgroup of DIS07:

http: //indico. cern. ch/contributionDisplay . py? contribId=276\&sessionId=5\&conf Id=9499.

[2] Slides: See Wu-Ki Tung, talk in the Structure Function workgroup in these proceedings: http://indico. cern. ch/contributionDisplay py? contribId=189\&sessionId=8\& conf Id=9499.

[3] W. K. Tung, H. L. Lai, A. Belyaev, J. Pumplin, D. Stump and C. P. Yuan, JHEP 0702, 053 (2007).

[4] See also C.-P. Yuan, talk in the Electroweak work group in these proceedings: http://indico. cern. ch/contributionDisplay . py? contribId=120\&sessionId=9\& conf Id=9499.

[5] H. L. Lai, P. Nadolsky, J. Pumplin, D. Stump, W. K. Tung and C. P. Yuan, JHEP 0704, 089 (2007).

[6] J. Pumplin, H. L. Lai and W. K. Tung, Phys. Rev. D 75, 054029 (2007). 